\title{
Globally coupled multiattractor maps: Mean field dynamics controlled by the number of elements
}

\author{
Imre M. Jánosi ${ }^{1,2,3}$ and Jason A. C. Gallas ${ }^{1,3}$ \\ ${ }^{1}$ Instituto de Física, Universidade Federal do Rio Grande do Sul, 91501-970 Porto Alegre, Brazil \\ ${ }^{2}$ Department of Physics of Complex Systems, Eötvös University, Múzeum körút 6-8, H-1088 Budapest, Hungary \\ ${ }^{3}$ Höchstleistungsrechenzentrum (HRLZ), Forschungszentrum Jülich, D-52425 Jülich, Germany
}

(Received 21 April 1998)

\begin{abstract}
We report an investigation of a system of $N$ globally coupled maps able to support multiattractors. For these systems the mean field dynamics is controlled by the number of elements in the initial partition of each basin of attraction. This behavior is in strong contrast with coupled systems of maps with a single attractor, where the mean field dynamics is usually simple for weak couplings. In spite of the increased local complexity, the global dynamics can be reduced to a simple two-dimensional map up to the first bifurcation point of the coexisting attractors. [S1063-651X(99)50601-6]

PACS number(s): 05.45.- a
\end{abstract}

Coupled map lattices (CML) provide perhaps the most popular type of complex dynamical systems for studying the generic properties and basic mechanisms of spatiotemporal chaos [1]. There are two extreme classes of CML's which differ fundamentally in their behavior. The first type is constituted by systems with short-range (typically nearestneighbor) interaction, and low-dimensional (1D, 2D) lattice architecture $[1,2]$. Its main feature is a fast decay of spatial correlations that leads to the convergence of statistical averages for $N \rightarrow \infty$. The second class is constituted by systems with global interactions, where the basic model introduced and studied extensively by Kaneko [2-4] is the globally coupled map lattice (GCML):

$$
x_{i}(n+1)=(1-\epsilon) f\left[x_{i}(n)\right]+\frac{\epsilon}{N} \sum_{j=1}^{N} f\left[x_{i}(n)\right]
$$

Here $n$ is a discrete time time variable, $f(x)$ is a map prescribing the local dynamics, and $i=1, \ldots N$ is the index identifying the elements. An analogy with globally coupled oscillator systems is pertinent [5]. In globally coupled systems, the absence of relaxation towards statistical equilibrium is one of the principal differences from the case of local coupling. That such a behavior is a consequence of longrange coupling was convincingly proven by Chaté and Manneville [6]: They found that in locally coupled highdimensional lattices, where the coordination number can be very large, spatiotemporal chaos takes the form of nontrivial collective behavior without stationary probability distributions.

By far, the most extensively studied "standard model" of GCML is based on the logistic map $f(x)=1-a x^{2}(x$ $\in[-1,1], a \in[0,2])$ as the local element in Eq. (1). The rich dynamics of this system is relatively well-known from numerical simulations $[2,3,7]$, even some rigorous analytical results have been recently obtained for the weak coupling limit [8]. A few extensions of the standard model by introducing heterogeneity [9] or noise [10] also attracted recent interest as realistic models of complex phenomena in physics $[1,5,7]$. Here we introduce an extension that reveals a striking feature: The partition of initial configurations $\left\{x_{i}(n=0)\right\}$ can play the role of control parameters in determining the global dynamics.

Similar to GCMLs, other complex systems can also possess a large number of coexisting attractors, like the mechanical rotor model studied in detail by Feudel et al. [11]. The common property of such systems is the so-called multistability, i.e., the existence of several different long term asymptotic states. Examples can be given from many fields, such as neuroscience [12], chemistry [13], optics [14], or condensed matter physics [15]. Our GCML proposed here can be considered as a prototype of multistable systems, and its simplicity can help a lot in the understanding of multiattractor dynamics.

The local map for Eq. (1) in our investigations is the restricted quartic map which is simply the second iterate of the logistic map:

$$
f(x)=1-a\left(1-a x^{2}\right)^{2} .
$$

The generic quartic map $f(x)=1-a\left(1-b x^{2}\right)^{2}$ has a single variable but two control parameters, and has a very complex stability domain structure, investigated in detail by one of us [16]. The restricted form of Eq. (2) preserves the basic bifurcation structure of the logistic map in parameter space, however, in sharp contrast with the logistic case, it displays two coexisting stable attractors (Fig. 1). The boundaries of the basins of attraction are given by the unstable fixed points $\pm(\sqrt{1+4 a}-1) / 2 a$. Internal crisis [17] occurs when the unstable fixed points coincide with the attractor, resulting in the "explosion" of the chaotic amplitude (see Fig. 1, at $a_{b m}$ $\approx 1.54365 \ldots$, which is also the band merging point for the logistic map). For parameters $a>a_{b m}$ the two attractors seem to be identical, i.e., numerically indistinguishable, but mathematical continuity ensures that individual trajectories starting from different initial values cannot overlap.

The separation of the basins of attraction obviously suggests that the mean field dynamics should depend strongly on the initial configurations. Indeed, the GCML with quartic maps can be forced to follow for a while either the top or the bottom attractor by a suitable preparation of the initial val- 


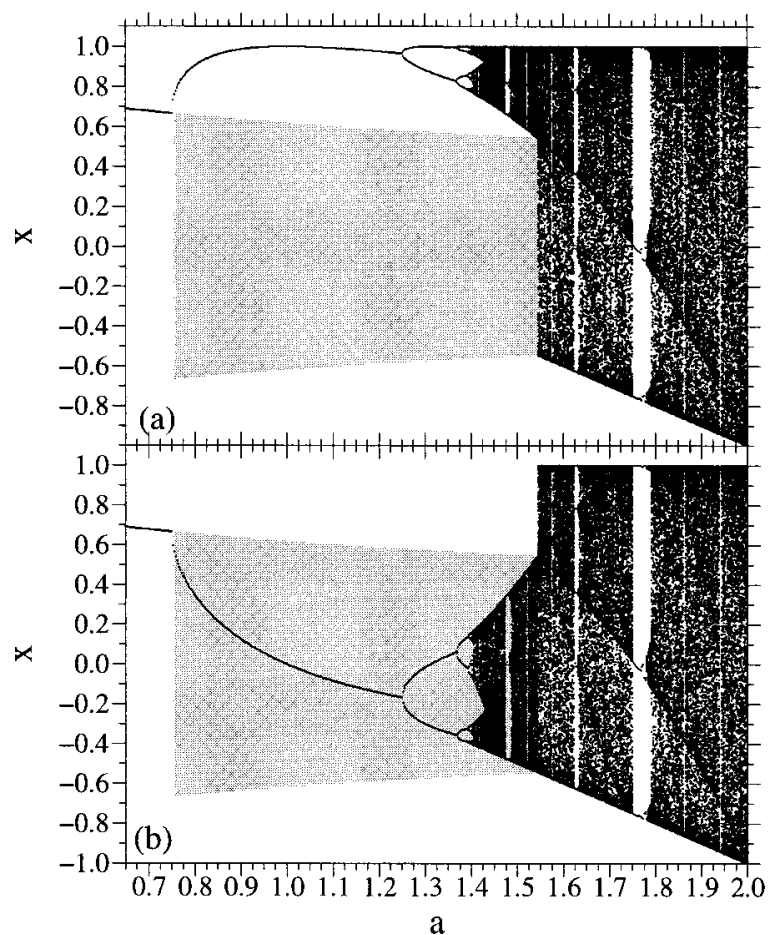

FIG. 1. Bifurcation diagram for the single quartic map Eq. (2). At each of the $a$ parameters, 300 values are plotted after discarding the first 50000 iterations. White and gray regions show the basins of attraction for (a) the "top," and (b) the "bottom" attractors, respectively.

ues; see Fig. 2. Here the mean field $h(n)$ $=(1 / N) \sum_{i=1}^{N} f\left[x_{i}(n)\right]$ and the average maximum Lyapunov exponent $\Lambda=(1 / N) \sum_{i=1}^{N} \lambda_{i}$ are plotted for coupled logistic and quartic maps too [18-20]. We could identify approximately the same dynamical ranges for the coupled quartic maps (coherent phase, ordered phase, partially ordered or glassy phase, and standard chaotic phase), as described in details for logistic maps $[2,7,10]$. In Fig. 2, arrows indicate where the first clustering instabilities arise, next we analyze this question in more detail.

Clustering is one of the most common phenomena in GCML [7,21-23]. The critical stability condition of a coherent state, where all of the elements are moving fully synchronized, is explicitly given [21] as $\lambda+\ln (1-\epsilon)<0$, where $\lambda$ is the Lyapunov exponent of the elementary map at the given parameter $a$. Note that this condition is generally valid for any coherent state, whatever its motion, periodic or chaotic. If the stability condition is not fulfilled, the system splits into a given number of clusters, where in each cluster the state variable of the members is identical. The first clustering instability in coupled logistic maps is fixed by the parameters $a$ and $\epsilon$ [Fig. 2(a)], but not the size of the clusters. Different initial configurations can result in different number of elements in the two clusters [23]. In a two-cluster state, the global dynamics can be reduced to a simple two-dimensional map [21]; however, reduction is possible only after the system has fallen onto two clusters.

The situation is rather different with quartic maps, since they support multiattractor local dynamics. Map (2) has two coexisting attractors with well defined basins, a given initial configuration determines the first clustering instability; see

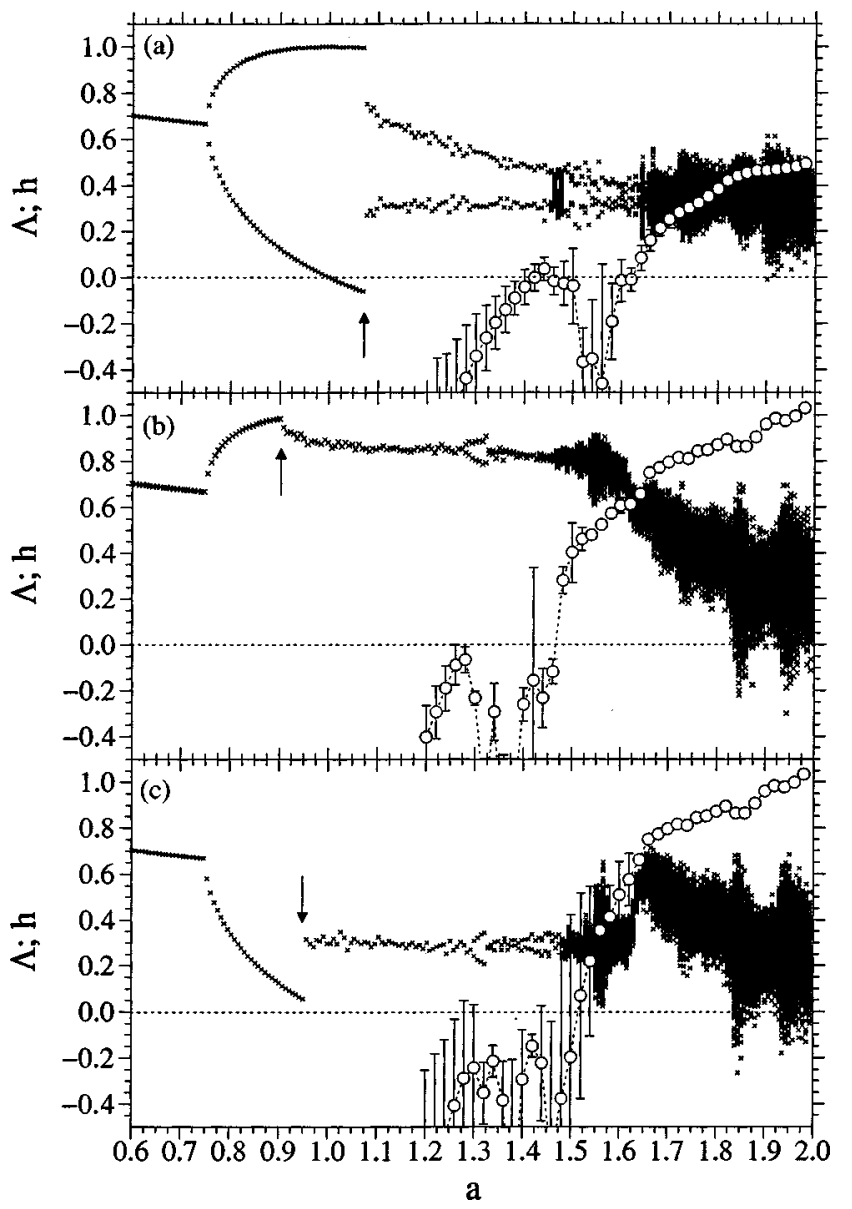

FIG. 2. Average Lyapunov exponent $\Lambda(\bigcirc)$ and the mean field $h$ $(\times)$ as a function of the dynamical parameter $a$, for $\epsilon=0.1$ and $N$ $=1600$. Five hundred values of $h$ are plotted after discarding 50000 iterations. The average value and the standard deviation for $\Lambda$ were determined in 50000 steps after discarding 50000 initial iterations. Arrows indicate the first clustering instability. (a) Logistic maps, the initial values were uniformly distributed random numbers in the range of $[0,1]$; (b) quartic maps, initial random values are in $[0.5,1]$; (c) quartic maps, random initial values in $[0,1]$.

Fig. 3. Furthermore, the following reduced two-dimensional map [21] gives a very good approximation for the collective dynamics:

$$
\begin{aligned}
& X_{n+1}^{\uparrow}=\left(1-\epsilon_{\downarrow}\right) f\left(X_{n}^{\uparrow}\right)+\epsilon_{\downarrow} f\left(X_{n}^{\downarrow}\right), \\
& X_{n+1}^{\downarrow}=\left(1-\epsilon_{\uparrow}\right) f\left(X_{n}^{\downarrow}\right)+\epsilon_{\uparrow} f\left(X_{n}^{\uparrow}\right) .
\end{aligned}
$$

Here $X_{n}^{\uparrow}$ and $X_{n}^{\downarrow}$ represent the clusters on the top ( $\left.\uparrow\right)$ and the bottom $(\downarrow)$ attractors at time-step $n$, and $\epsilon_{\uparrow}=k \epsilon$ and $\epsilon_{\downarrow}$ $=(1-k) \epsilon$ are the effective coupling constants for a partitioning $k=K / N$. The mean field is then simply $h=k X_{n}^{\uparrow}$ $+(1-k) X_{n}^{\downarrow}$. In Fig. 4 we show how the first clustering parameter $a_{c}$ depends on the partitioning $k$ for several coupling constants $\epsilon$ and system sizes $N$. There is a critical ratio $k_{c}$, which separates the common attractor for $a<a_{c}$ : At $k$ $<k_{c}$, the coherent state settles on the bottom attractor, while at $k>k_{c}$ on the top attractor. Note that the weight of the two attractors is rather different. The critical ratio $k_{c}=k_{c}(\epsilon)$ $\approx 0.57-0.62$ and the higher slopes at $k<k_{c}$ show that the bottom attractor represents a "deeper minimum," by using 


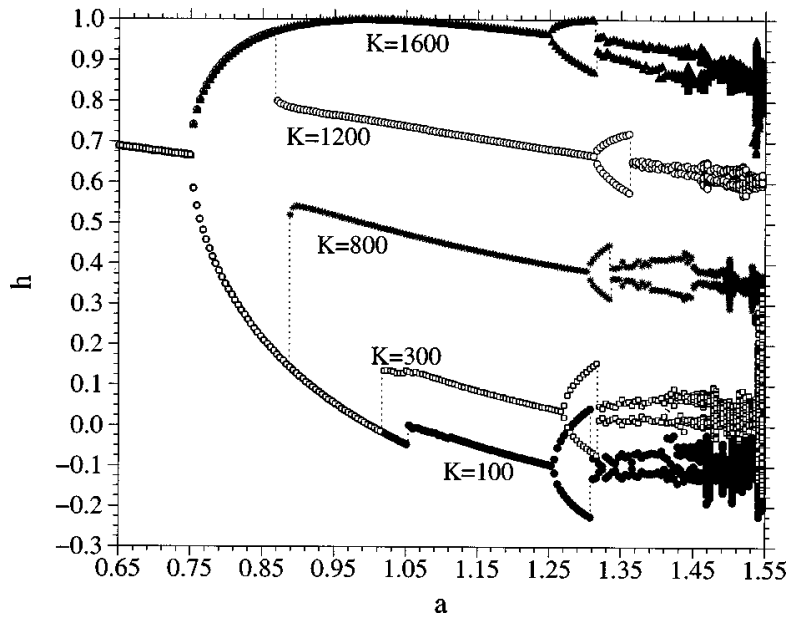

FIG. 3. Mean field bifurcation diagram for coupled quartic maps in the periodic range. Different initial configurations were prepared by dividing the sites into two clusters: $K$ sites (see the labels) out of $N=1600$ had random starting values in the basin of the top attractor, $N-K$ in the basin of the bottom attractor (see Fig. 1). After discarding 50000 iterations, 100 subsequent values of $h$ are plotted for each $a$.

an analogy from two-state systems. Since the mean field attractor from a randomized initial configuration is always different from that of the elementary map, the results of the direct simulations show a slight systematic deviation from the reduced map representation [Eq. (3)], see the nonlinear curves at $k<k_{c}$ for the coupled maps (symbols in Fig. 4). For the same reason the functional form of $a_{c}(k ; \epsilon)$ is not universal, although a partial data collapse can be obtained for $k>k_{c}$ by the transformation $a_{c} \rightarrow\left(a_{c}-a_{2}\right) / \epsilon$, where $a_{2}$ $=0.75$ is the first bifurcation point for the logistic map (see Fig. 4, inset).

The two-dimensional reduced map approximation breaks down at the second clustering instability, which occurs at $a$

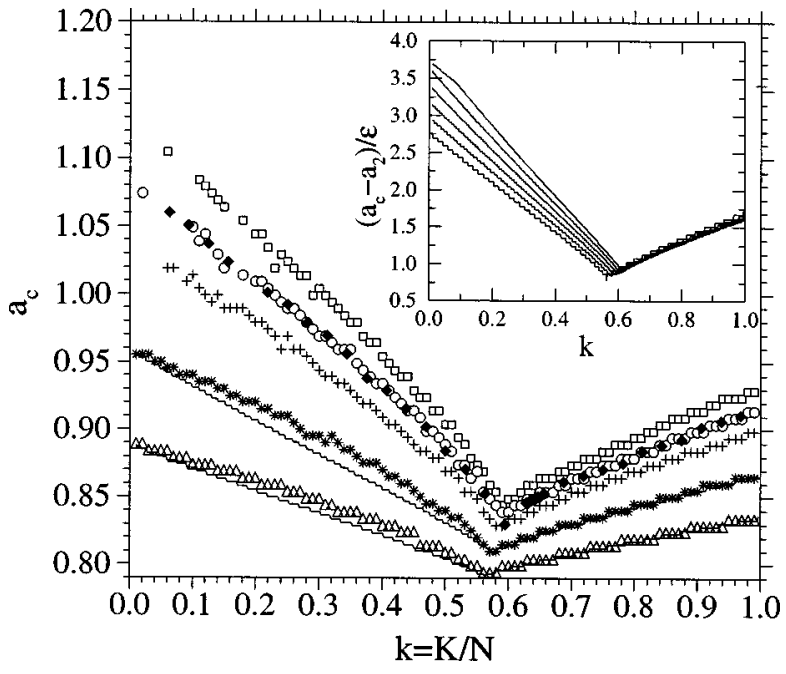

FIG. 4. First clustering parameter $a_{c}$ as a function of partition $k=K / N$, where $K$ elements from the total of $N$ have initial value in the basin of the top attractor. Symbols denote lattice simulations with $\epsilon=0.05, N=500(\triangle) ; \epsilon=0.07, N=500(*) ; \epsilon=0.09, N$ $=800(+) ; \epsilon=0.1, N=500(\bigcirc) ; \epsilon=0.1, N=1500(\diamond) ; \epsilon=0.11$, $N=500(\square)$. Solid lines are obtained by the reduced map (3). The inset shows a partial data collapse; see the text.

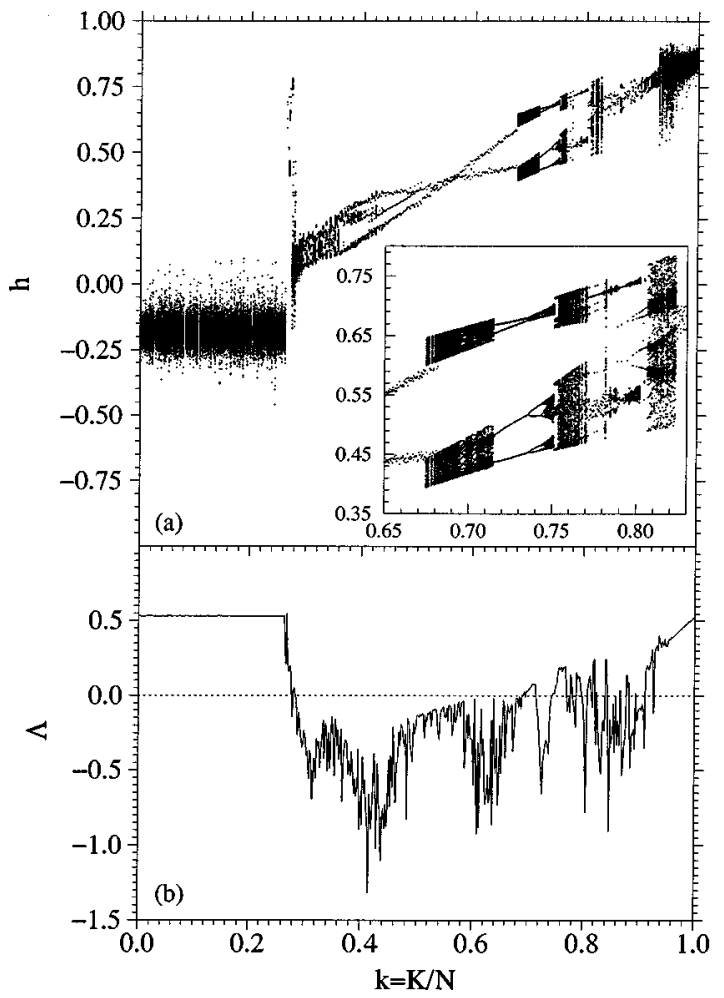

FIG. 5. Bifurcation diagram for the mean field $h$ and average Lyapunov exponent $\Lambda$ as a function of partition $k=K / N$, where $K$ elements from the total of $N=5000$ have random initial values $\in[0.6,0.8]$; the rest is $\in[0,0.5]$. For each case, 50000 initial iterations were discarded and the next 200 values for $h$ are plotted. The inset shows a zoomed region. $\Lambda$ is obtained as in Fig. 2. The other parameters are $a=1.58$ and $\epsilon=0.25$.

$>1.3$ (cf. Fig. 3). A higher order cluster expansion, e.g., a similar one which was successfully developed for local coupling in Ref. [24], could provide a proper description for small number of clusters, but in the chaotic range at weak couplings the number of clusters can be comparable with the number of elements itself. In our system, however, there is a rather wide parameter range of local chaos with simple global dynamics. Next we analyze this case.

It is known from studies with coupled logistic maps that different partitionings even in a two-cluster state can result in different dynamics from periodic to chaotic, considering the motion of the single elements in the clusters [7,21-23]. The mean field dynamics, however, remains usually simple in the sense that the amplitude distribution of the mean field fluctuations is Gaussian [4]. The width of the Gaussian is decreasing with $N$ up to a given critical size $N_{c}$, where the emergence of coherences leads to the apparent violation of the law of large numbers [25], discussed in detail in the literature and explained by Pikovsky and Kurths [26].

The success of the reduced two-dimensional map description seems to suggest that the system of globally coupled quartic maps has a simpler dynamics than a GCML with logistic maps, at least in the parameter range where the basins of attraction are well separated. However, as we have shown already, the two attractors of the quartic map do not have equal weight in the formation of the global dynamics. This asymmetry holds also in parameter ranges where the internal dynamics of the top and the bottom is more compli- 
cated than a simple fixed point. Thus we expect that the collective attractors realized at the top and at the bottom can be different depending on the global coupling and the number of sites $N$. Here we illustrate that this is really the case.

Figure 5 shows a representative bifurcation diagram for the mean-field dynamics as a function of partitioning of the initial configurations. We emphasize that the separation of the basins of attraction for the collective attractors is very good, yet, riddling [19] begins at around $a \approx 1.74$ for the given coupling $\epsilon=0.25$ [20]. Accordingly, the global dynamics can be chaotic, pure periodic, and any mixture of them, and the only apparent control parameter is the number of elements in the different basins of attraction.
In summary, we have shown that the global dynamics of our simple coupled multiattractor system can be effectively controlled by the partitioning of initial configurations in the different basins of attraction. We emphasize that this partitioning does not require a very precise preparation of initial values, in this sense the dynamics is not sensitive for small amplitude noise, like other multistable systems [11].

I.M.J. thanks FAPERGS, Porto Alegre, for the travel Grant No. 200.644/98-67. This work was partially supported by the Hungarian National Science Foundation (OTKA) under Grant No. F014967. I.M.J. thanks the Foundation for Research and Higher Education for financial support. J.A.C.G. thanks CNPq, Brazil, for partial support.
[1] Theory and Applications of Coupled Map Lattices, edited by K. Kaneko (Wiley, New York, 1993).

[2] K. Kaneko, Prog. Theor. Phys. 89, 263 (1989).

[3] K. Kaneko, Phys. Rev. Lett. 63, 219 (1989); Physica D 41, 38 (1990).

[4] K. Kaneko, Physica D 55, 368 (1992).

[5] P. Hadley, M. R. Beasley, and K. Wiesenfeld, Phys. Rev. B 38, 8712 (1988); K. Nakagawa and Y. Kuramoto, Prog. Theor. Phys. 89, 313 (1989); S. H. Strogatz et al., Physica D 36, 23 (1989); K. Wiesenfeld et al., Phys. Rev. Lett. 65, 1749 (1990); H. Sompolinsky, D. Golomb, and D. Kleinfeld, Phys. Rev. A 43, 6990 (1991).

[6] H. Chaté and P. Manneville, Europhys. Lett. 17, 291 (1992); Prog. Theor. Phys. 87, 1 (1992); H. Chaté, A. Lamaître, P. Marcq, and P. Manneville, Physica A 224, 447 (1996).

[7] A. Crisanti, M. Falcioni, and A. Vulpiani, Phys. Rev. Lett. 76, 612 (1996).

[8] S. V. Ershov and A. B. Potapov, Physica D 106, 9 (1997).

[9] T. Shibata and K. Kaneko, Europhys. Lett. 38, 417 (1997).

[10] K. Kaneko, Phys. Rev. Lett. 78, 2736 (1997).

[11] U. Feudel et al., Phys. Rev. E 54, 71 (1996); Chaos 7, 597 (1997); Chaos Solitons Fractals 9, 1771 (1998).

[12] S. Schiff et al., Nature (London) 370, 615 (1994); J. Foss et al., Phys. Rev. Lett. 76, 708 (1996).

[13] P. Marmillot, M. Kaufman, and J. F. Hervagault, J. Chem. Phys. 95, 1206 (1991); K. L. C. Hunt et al., ibid. 96, 7019
(1992).

[14] M. Brambilla et al., Phys. Rev. A 43, 5090 (1991); 43, 5114 (1991).

[15] F. Prengel, A. Wacker, and E. Schöll, Phys. Rev. B 50, 1705 (1994); B. S. Ning, ibid. 51, 11221 (1995).

[16] J. A. C. Gallas, Phys. Rev. E 48, R4156 (1993); Physica A 202, 196 (1994); 211, 57 (1994); Appl. Phys. B: Lasers Opt. B60, S-203 (1995).

[17] C. Grebogi, E. Ott, and J. A. Yorke, Phys. Rev. Lett. 48, 1507 (1982); Physica D 7, 181 (1983).

[18] Since there is an analytical expression for the local dynamics, Eq. (2), exponents are obtained by summing the log of the magnitude of the derivative of the map along the trajectory

[19] J. C. Alexander et al., Int. J. Bifurcation Chaos Appl. Sci. Eng. 2, 795 (1992); E. Ott et al., Phys. Rev. Lett. 71, 4134 (1993).

[20] I. M. Jánosi and J. A. C. Gallas (unpublished).

[21] K. Kaneko, Phys. Rev. Lett. 63, 219 (1989).

[22] K. Kaneko, J. Phys. A 24, 2107 (1991).

[23] F. Xie and G. Hu, Phys. Rev. E 56, 1567 (1997).

[24] A. Lemaître, H. Chaté, and P. Manneville, Phys. Rev. Lett. 77, 486 (1996).

[25] K. Kaneko, Phys. Rev. Lett. 65, 1391 (1990); G. Perez, C. Pando-Lambruschini, S. Sinha, and H. Cerdeira, Phys. Rev. A 45, 5469 (1992).

[26] A. S. Pikovsky and J. Kurths, Phys. Rev. Lett. 72, 1644 (1994); Physica D 76, 411 (1994). 